Research Article

\title{
Exploring the Potential Mechanism of Tang-Shen-Ning Decoction against Diabetic Nephropathy Based on the Combination of Network Pharmacology and Experimental Validation
}

\author{
Jiajun Liang $\mathbb{D}$, Jiaxin He,,$^{1,2}$ Yanbin Gao $\mathbb{D}^{1,2}$ and Zhiyao Zhu $\mathbb{D}^{1,2}$ \\ ${ }^{1}$ School of Traditional Chinese Medicine, Capital Medical University, Beijing 100069, China \\ ${ }^{2}$ Beijing Key Laboratory of TCM Collateral Disease Theory Research, Beijing 100069, China \\ Correspondence should be addressed to Yanbin Gao; gyb@ccmu.edu.cn and Zhiyao Zhu; 20120103@ccmu.edu.cn
}

Received 10 May 2021; Accepted 11 August 2021; Published 9 September 2021

Academic Editor: Amjad Iqbal

Copyright (C) 2021 Jiajun Liang et al. This is an open access article distributed under the Creative Commons Attribution License, which permits unrestricted use, distribution, and reproduction in any medium, provided the original work is properly cited.

\begin{abstract}
Background. Diabetic nephropathy (DN) has become one of the leading causes of the end-stage renal disease (ESRD). Tang-ShenNing (TSN) decoction, an effective Traditional Chinese formula for DN, can improve the renal function and inhibit renal fibrosis in DN. However, its potential mechanism is still unexplored. Methods. A network pharmacology approach was employed in this study, including screening for differential expressed genes of DN (DN-DEGs), protein-protein interaction (PPI) network analysis, and GO and KEGG enrichment analysis. Besides, a rat model was established to verify the potential effect of TSN in DN. Results. Twenty-three TSN-related DN-DEGs targets were identified. These genes were associated with decreased glomerular filtration rate (GFR) DN. The enrichment analysis suggested that the inhibition of renal fibrosis and inflammation through growth factors and chemokines is the potential mechanism through which TSN improves DN. TSN reduced renal fibrosis and improved pathological damage in the kidney in vivo through the regulation of GJA1, CTGF, MMP7, and CCL5, which are genes associated with ECM deposition. Conclusion. This study revealed that TSN improves DN through a multicomponent, multitarget, and multipathway synergy. We provide a scientific basis for potential targets for TSN use to treat DN, yet further experimental validation is needed to investigate these targets and mechanisms.
\end{abstract}

\section{Introduction}

Diabetic nephropathy (DN), a severe microvascular complication of long-term diabetes mellitus (DM), is present in $40 \%$ of diabetic patients [1]. DN leads to chronic progressive renal damage, accompanied by increased urinary proteins and decreased glomerular filtration rate (GFR). Indeed, DN becomes a leading cause of progression to end-stage renal disease (ESRD) in DM patients [2,3]. KDOQI proposed that the term "diabetic nephropathy" should be replaced by "diabetic kidney disease" (DKD), which applies to a kidney disease that is caused by diabetes, while DN can be diagnosed only after the histopathological confirmation. The pathological progression of DN includes glomerular basement membrane thickening, mesangial matrix hyperplasia, and glomerulosclerosis [4]. DN's pathogenesis is complex and includes alterations in renal hemodynamics, disturbances in glucolipid metabolism, the action of various cytokines, and activation of the Renin-AngiotensinAldosterone System (RAAS). RAAS inhibitors have some benefit in the treatment of DN. However, according to clinical studies, when patients were treated with dual RAAS blocking, not only is it less effective in the long term, but patients were also more likely to suffer from risky events like acute kidney injury or hyperkalemia $[5,6]$. Therefore, it is essential to identify novel therapeutic schedule for DN.

In China, Traditional Chinese Medicine (TCM) has a long history of treating $\mathrm{DM}$ and $\mathrm{DN}$ and provides renal protection for $\mathrm{DN}$ through different herbal combinations [7]. Developed by Yanbin Gao, a TCM expert, Tang-Shen- 
Ning decoction (TSN) is an empirical formula to treat DN, consisting of Astragalus membranaceus (Fisch.) Bunge (Huang Qi in Chinese, HQ, 15 g), Euryale ferox Salisb. ex DC (Qian Shi in Chinese, QS, $15 \mathrm{~g}$ ), Rosa laevigata Michx (Jin Ying $Z i$ in Chinese, JYZ, $15 \mathrm{~g}$ ), Rheum officinale Baill ( $\mathrm{Da}$ Huang in Chinese, DH, 6g), and Ligusticum chuanxiong Hort (Chuan Xiong in Chinese, CX, $12 \mathrm{~g}$ ). According to a double-blinded control clinical trial on DN patients, the use of TSN led to reductions in 24-hour urine albumin excretion rate ( $24 \mathrm{~h}$ UAER) compared to control group. Besides, patients in the TSN treatment group showed improvements in serum SOD, MDA, and hs-CRP compared to the control group, indicating that TSN is a safe and effective medicine for the treatment of early DN and improving the inflammatory and oxidative stress status in DN [8]. It was also shown that TSN treatment in DN mice decreased $24 \mathrm{~h}$ UAER, serum creatinine, and blood urea nitrogen. Furthermore, TSN activated the $\mathrm{Wnt} / \beta$-catenin pathway, reversed the podocyte epithelial-mesenchymal transition (EMT), reduced the expression of fibroblast-specific protein 1 (FSP-1) and collagen I, and alleviated kidney damage in DN mice [9]. However, the effect of TCM is multitargeted and affects multiple pathways; therefore, the potential mechanism of TSN in DN still needs to be investigated.

Currently, significant developments are taking place in the field of systems biology, in which network pharmacology is considered an important tool for drug discovery. Network pharmacology aims to describe a biological system using a network structure, switching the research paradigm to "network-targeted, multicomponent therapy." This research model has similarities with TCM due to the synergistic mechanism of multicomponent therapeutic approaches that affect multiple pathways and therefore underlying mechanisms of TCM can be elucidated through network pharmacology $[10,11]$. Therefore, we performed a systems biology-based approach to explore the underlying mechanisms of TSN in DN (Figure 1) as a guidance for further research.

\section{Materials and Methods}

2.1. Screening of TSN Compounds with Potential Biological Activity. TCMSP database (http://tcmspw.com/tcmsp.php) [12] was searched to obtain the candidate ingredients of the fives herbs contained in TSN. Then, compounds with oral bioavailability $(\mathrm{OB}) \geq 30 \%$ and drug-likeness (DL) $\geq 0.18$ were considered as potential bioactive ingredients $[11,13]$.

2.2. Target Screening. The TCMSP, PubChem database (https://pubchem.ncbi.nlm.nih.gov/) [14], and STITCH platform (http://stitch.embl.de/cgi/network.pl) [15] were used to search the targets of potential bioactive ingredients in TSN. In the PubChem database, targets were collected from the following four sections: Chemical-Gene Co-Occurrences in Literature, Protein Bound 3D Structures, DrugGene Interactions, and BioAssay Results. The threshold score was set to 0.9 to filter targets predicted by STITCH.
2.3. Identification of Differentially Expressed Genes (DEGs). Gene expression data from $9 \mathrm{DN}$ patients and 13 normal human glomeruli in GSE30528 Genest were downloaded from GEO database (https://www.ncbi.nlm.nih.gov/geo/), which were firstly contributed by Woroniecka et al. [16]. DEGs of glomeruli from DN patients and normal samples were obtained using the GEO2R online tool by setting | $\log \mathrm{FC} \mid>1.5$ and $p<0.05$. The gene expression was considered downregulated when the assay results indicated $\log \mathrm{FC}<0$; if $\operatorname{LogFC}>0$, the gene expression was upregulated.

2.4. Network Construction. Networks were constructed using Cytoscape v3.8.0 [17] as follows: (1) component-target network, (2) protein-protein interaction (PPI) network for DEGs, (3) component-DEGs network, and (4) target-signaling pathway network.

2.5. PPI Network and Topological Analysis. TSN-related DEGs in DN (DN-DEGs) were identified as genes up- or downregulated in both TSN treatment and DN. The PPI network of TSN-related DN-DEGs was constructed using Bisogenet in Cytoscape. Three topology parameters, namely, degree, betweenness centrality, and closeness centrality, were chosen to analyze the topology of the network diagram, which reflected the topological importance of the nodes. Nodes with corresponding parameters greater than 2 times the median were selected to finally obtain the core targets of the PPI network [18]. "Degree" indicates how many edges were connected to a node; "betweenness centrality" is the times a node appears on the shortest path versus the total number of paths; "closeness centrality" measures the closeness of a node by calculating the distance of the shortest path from that node to the others [19].

2.6. Gene Ontology (GO) and KEGG Pathway Enrichment Analysis. The hub targets in the PPI network were analyzed using Metascape (http://metascape.org/gp/index.html) [20]. The enrichment results with $p<0.05$ were ranked, and those with $p<0.01$ were considered significantly enriched. Finally, the biological processes (BP), molecular functions (MF), cellular components (CC), and signaling pathways were identified.

2.7. Correlation of TSN-Related DN-DEGs with GFR. Nephroseq v5 (http://v5.nephroseq.org) is an online platform to mine comprehensive nephropathy gene expression datasets to identify markers of disease progression by correlating renal genetic phenotypes with known disease phenotypes [21]. Nephroseq v5 platform was applied to analyze the correlation between TSN-related DN-DEGs and GFR. TSN-related DN-DEGs were searched and the dataset Woroniecka Diabetes Glom, which is linked to GSE30528, was selected. 


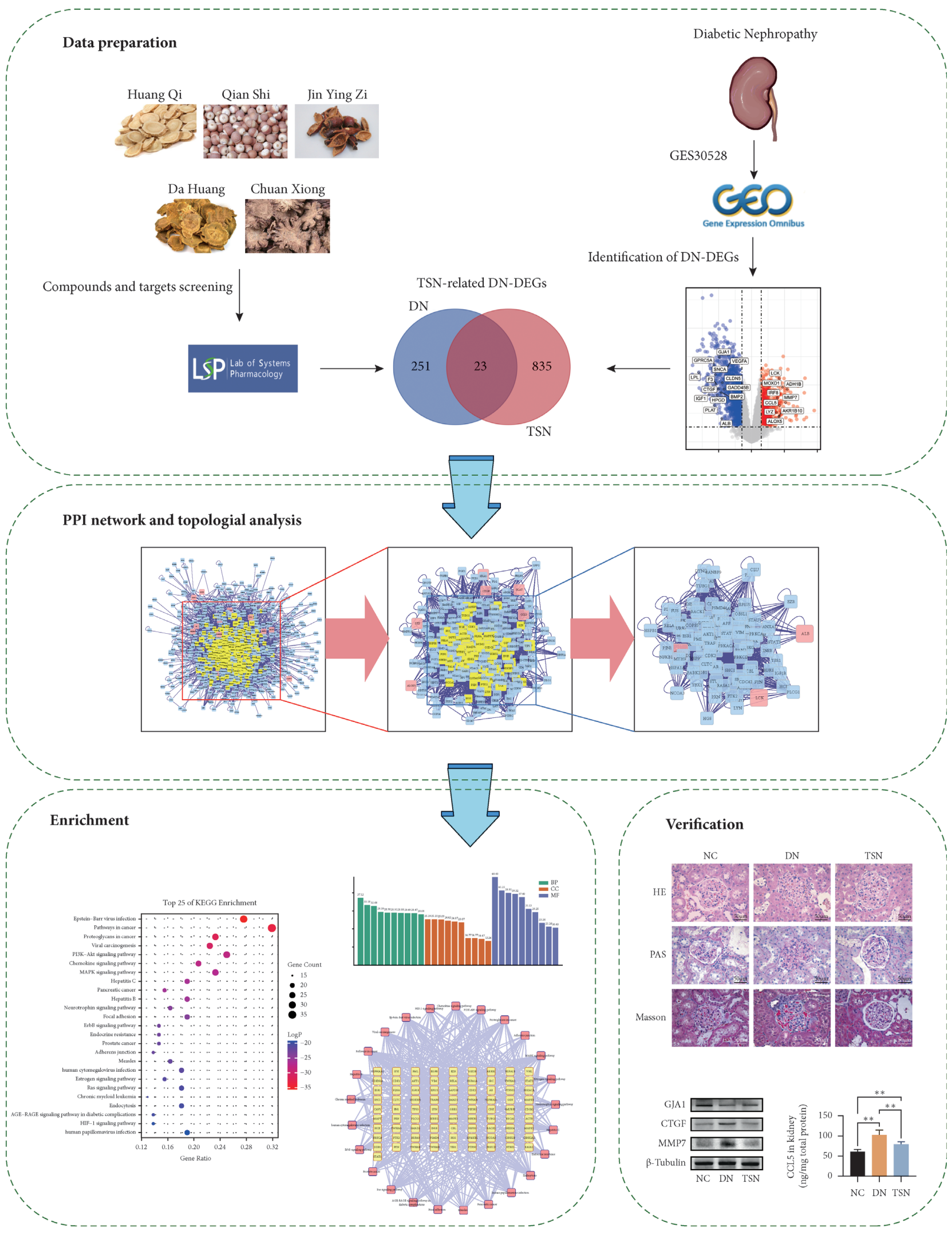

FIGURE 1: The schematic diagram of network pharmacological study of TSN for DN. 


\subsection{Experimental Validation}

2.8.1. Reagents. Streptozocin (STZ) was purchased from Sigma. (RANTES/CCL5) ELISA kit (CSB-E07398r) was purchased from Cusabio Biotech. Mouse monoclonal antiCTGF antibody (Cat\#: SC-365970) was purchased from Santa Cruz Biotechnology. Rabbit polyclonal anti-MMP7 antibodies (Cat\#: 3801) and Rabbit polyclonal anti-GJA1 antibodies (Cat\#: 3512) were purchased from Cell Signaling Technology. Mouse monoclonal anti- $\beta$-Tubulin antibody (Cat\#: C1340) was purchased from APPLYGEN. Donkey Anti-Mouse IgG was purchased from Proteintech. Goat Anti-Rabbit IgG was purchased from LabLead.

2.8.2. Animals and Models. Eighteen male Sprague Dawley rats (180-220 g) were purchased from Weitonglihua (Beijing, China). Rats were fed and given water ad libitum in an SPF environment. All procedures were approved by the Animal Experiments and Experimental Animal Welfare Committee of Capital Medical University.

Rats were randomly distributed, after one week of acclimatization, into normal control (NC, $n=6$ ), DN group (DN, $n=6$ ), and TSN group (TSN, $n=6$ ). The DN rat model used in both DN and TSN groups was established as described previously [22]. Briefly, rats received intraperitoneal injections of streptozotocin (STZ, $55 \mathrm{mg} / \mathrm{kg}$ ) and a high-fat diet (10\% lard, $20 \%$ sucrose, $2.5 \%$ cholesterol, $0.5 \%$ sodium cholate, and $67 \%$ basic feed). Rats in NC were injected with the same dose of sodium citrate buffer and fed with a normal diet (12\% fat, $28 \%$ protein, and $60 \%$ carbohydrate). After 7 days, random blood glucose (RGB) was measured in all rats in the diabetic group, and those rats with RBG levels above $16.7 \mathrm{mmol} / \mathrm{L}$ for three consecutive days were considered diabetic. Diabetic rats were randomly distributed into DN (DN, $n=6$ ) and TSN (TSN, $n=6$ ) groups. Rats in TSN group were administered $20 \mathrm{~g} / \mathrm{kg}$ TSN orally every day while rats in $\mathrm{NC}$ and DN were given the same volume of normal saline instead. The rats were euthanized after 12 weeks of treatment. Fresh kidneys were dissected and preserved under $-80^{\circ} \mathrm{C}$ for further experiments.

2.8.3. Histological Analysis. Formalin-fixed kidney tissues were embedded in paraffin and stained with hematoxylineosin (HE), Periodic Acid-Schiff (PAS), and Masson. The slices were analyzed and captured using a light microscope (Leica, DM4B) under $\times 400$ magnification.

2.8.4. ELISA Assay. CCL5 levels in the rat kidneys were measured using ELISA kit (Cusabio, Wuhan, China) according to the instructions provided.

2.8.5. Western Blot Analysis. Kidney tissues were lysed and the supernatant was collected after centrifugation. After homogenization, protein symbol was separated using $10 \%$ SDS-PAGE electrophoresis and electrotransferred to PVDF membranes. Membranes were blocked with 5\% non-fat milk and then incubated overnight at $4^{\circ} \mathrm{C}$ with the proper primary antibody for anti-GJA1 (Cell Signaling Technology, USA), CTGF (Santa Cruz Biotechnology, USA), MMP7 (Cell Signal Technology, USA), and $\beta$-Tubulin (APPLYGEN, China). After that, the membranes were incubated with corresponding secondary antibodies, including Donkey AntiMouse IgG (Proteintech, USA) and Goat Anti-Rabbit IgG (LabLead, China) at room temperature. Antigen-antibody immunoreactivity was visualized using electrochemiluminescence (ECL) reagents (Millipore, USA). Protein expressions were normalized according to the intensity of $\beta$-Tubulin and analyzed with Gelpro Analyzer software.

2.9. Statistical Analyses. Pearson's correlations between GFR and TSN-related DN-DEGs were performed using Nephroseq v5. Experimental data were presented as mean $\pm \mathrm{SD}$ and analyzed using Graphpad prism 9.00. One-way ANOVA was applied for comparison between more than two groups. $p<0.05$ means the comparison was statistically significant.

\section{Results}

3.1. Potential Bioactive Components and Targets in TSN. After screening with OB and DL value, 52 potential bioactive compounds were collected, including 20 from Huang Qi, 2 from Qian Shi, 16 from Da Huang, 7 from Jin Yin Zi, and 7 from Chuan Xiong and finally, 47 bioactive compounds were obtained by removing duplicates (Table 1). By searching these 47 components of TSN in TCMSP, PubChem, and STITCH databases, we obtained 858 corresponding potential targets. The Compound-Target network of TSN suggested potential synergistic effects of these herbs at shared targets (Figure 2).

3.2. DEGs Identified in DN. Glomerular gene expression data from GSE30528 were analyzed, resulting in 274 DEGs (Figure 3 and Supplementary Table S1), including 67 upregulated (represented by red plots) and 207 downregulated genes (blue plots).

3.3. DN-DEGs Related to TSN. After mapping the DN-DEGs to 858 potential TSN targets, 23 common genes were collected as critical effect targets of TSN in DN (Figure 4) and this information is shown in Table 2. The expression levels of common targets provided by the matrix file of GSE30528 are shown in Supplementary Fig. S1. A disease network including TCM compounds was constructed based on common targets (Figure 5), in which HQ contained the most active components associated with DN-DEGs, with a total of 7 targets, suggesting that HQ may be the most effective component in TSN. In addition, mairin (MOL000211) was related to the highest number of targets ( 7 in total), followed by quercetin (MOL000098) and hederagenin (MOL000296) (both with 5).

3.4. PPI Network Targets and Analysis. TSN-related DNDEGs were imported into Bisogenet in Cytoscape to generate the PPI network. The network consisted of 883 nodes 
TABLE 1: The potential bioactive compounds in TSN.

\begin{tabular}{|c|c|c|c|c|}
\hline Mol ID & Compounds & $\mathrm{OB}$ & $\mathrm{DL}$ & Herb \\
\hline MOL000211 & Mairin & 55.38 & 0.78 & HQ \\
\hline MOL000239 & Jaranol & 50.83 & 0.29 & HQ \\
\hline MOL000296 & Hederagenin & 36.91 & 0.75 & HQ \\
\hline MOL000033 & $\begin{array}{c}\text { (3S,8S,9S,10R,13R,14S,17R)-10,13-Dimethyl-17-[(2R,5S)-5-propan-2-yloctan-2-yl]- } \\
2,3,4,7,8,9,11,12,14,15,16,17 \text {-dodecahydro-1H-cyclopenta[a]phenanthren-3-ol }\end{array}$ & 36.23 & 0.78 & HQ \\
\hline MOL000354 & Isorhamnetin & 49.6 & 0.31 & HQ \\
\hline MOL000371 & 3,9-Di-O-methylnissolin & 53.74 & 0.48 & HQ \\
\hline MOL000374 & $5^{\prime}$-Hydroxyiso-muronulatol-2', $5^{\prime}$-di-O-glucoside & 41.72 & 0.69 & HQ \\
\hline MOL000378 & 7-O-Methylisomucronulatol & 74.69 & 0.3 & HQ \\
\hline MOL000379 & 9,10-Dimethoxypterocarpan-3-O- $\beta$-D-glucoside & 36.74 & 0.92 & HQ \\
\hline MOL000380 & (6aR,11aR)-9,10-Dimethoxy-6a,11a-dihydro-6H-benzofurano [3,2-c]chromen-3-ol & 64.26 & 0.42 & HQ \\
\hline MOL000387 & Bifendate & 31.1 & 0.67 & HQ \\
\hline MOL000392 & Formononetin & 69.67 & 0.21 & HQ \\
\hline MOL000398 & Isoflavanone & 109.99 & 0.3 & HQ \\
\hline MOL000417 & Calycosin & 47.75 & 0.24 & HQ \\
\hline MOL000422 & Kaempferol & 41.88 & 0.24 & $\begin{array}{l}\text { HQ, } \\
\text { JYZ }\end{array}$ \\
\hline MOL000433 & FA & 68.96 & 0.71 & $\begin{array}{l}\mathrm{HQ}, \\
\mathrm{CX}\end{array}$ \\
\hline MOL000438 & (3R)-3-(2-Hydroxy-3,4-dimethoxyphenyl)chroman-7-ol & 67.67 & 0.26 & HQ \\
\hline MOL000439 & Isomucronulatol- $7,2^{\prime}$-di-O-glucosiole & 49.28 & 0.62 & HQ \\
\hline MOL000442 & 1,7-Dihydroxy-3,9-dimethoxy pterocarpene & 39.05 & 0.48 & HQ \\
\hline MOL000098 & Quercetin & 46.43 & 0.28 & $\begin{array}{l}\text { HQ, } \\
\text { JYZ }\end{array}$ \\
\hline MOL002773 & Beta-carotene & 37.18 & 0.58 & QS \\
\hline MOL007180 & Vitamin-E & 32.29 & 0.7 & QS \\
\hline MOL001494 & Mandenol & 42 & 0.19 & $\begin{array}{l}\text { JYZ, } \\
\text { CX }\end{array}$ \\
\hline MOL000358 & Beta-sitosterol & 36.91 & 0.75 & $\begin{array}{l}\mathrm{JYZ}, \\
\mathrm{DH}\end{array}$ \\
\hline MOL005030 & Gondoic acid & 30.7 & 0.2 & JYZ \\
\hline MOL008622 & Methyl trametenolate & 42.88 & 0.82 & JYZ \\
\hline MOL008628 & $4^{\prime}$-Methyl-N-methylcoclaurine & 53.43 & 0.26 & JYZ \\
\hline MOL002280 & Torachrysone-8-O-beta-D-(6'-oxayl)-glucoside & 43.02 & 0.74 & $\mathrm{DH}$ \\
\hline MOL002281 & Toralactone & 46.46 & 0.24 & $\mathrm{DH}$ \\
\hline MOL002288 & Emodin-1-O-beta-D-glucopyranoside & 44.81 & 0.8 & $\mathrm{DH}$ \\
\hline MOL002293 & Sennoside D_qt & 61.06 & 0.61 & $\mathrm{DH}$ \\
\hline MOL002297 & Daucosterol_qt & 35.89 & 0.7 & $\mathrm{DH}$ \\
\hline MOL002303 & Palmidin A & 32.45 & 0.65 & $\mathrm{DH}$ \\
\hline MOL000471 & Aloe-emodin & 83.38 & 0.24 & $\mathrm{DH}$ \\
\hline MOL000554 & Gallic acid-3-O-(6'-O-galloyl)-glucoside & 30.25 & 0.67 & $\mathrm{DH}$ \\
\hline MOL000096 & $(-)$-Catechin & 49.68 & 0.24 & $\mathrm{DH}$ \\
\hline MOL002135 & Myricanone & 40.6 & 0.51 & $\mathrm{CX}$ \\
\hline MOL002140 & Perlolyrine & 65.95 & 0.27 & $\mathrm{CX}$ \\
\hline MOL002151 & Senkyunone & 47.66 & 0.24 & $\mathrm{CX}$ \\
\hline MOL002157 & Wallichilide & 42.31 & 0.71 & $\mathrm{CX}$ \\
\hline MOL000359 & Sitosterol & 36.91 & 0.75 & $\mathrm{CX}$ \\
\hline MOL002235 & Eupatin & 50.8 & 0.41 & $\mathrm{DH}$ \\
\hline MOL002251 & Mutatochrome & 48.64 & 0.61 & $\mathrm{DH}$ \\
\hline MOL002259 & Physciondiglucoside & 41.65 & 0.63 & $\mathrm{DH}$ \\
\hline MOL002260 & Procyanidin $\mathrm{B}-5,3^{\prime}$-O-gallate & 31.99 & 0.32 & $\mathrm{DH}$ \\
\hline MOL002268 & Rhein & 47.07 & 0.28 & $\mathrm{DH}$ \\
\hline MOL002276 & Sennoside E_qt & 50.69 & 0.61 & $\mathrm{DH}$ \\
\hline
\end{tabular}

9507 edges. The targets with "degree," "betweenness centrality," and "closeness centrality" above median values were selected as key targets. Details of the two screening and threshold setting are shown in Figure 6 and Supplementary Table S2. The screening results showed key underlying pathways of TSN in DN. A core PPI network consisting of 116 nodes and 1894 edges was constructed.
3.5. GO and KEGG Pathway Enrichment Analysis. Enrichment analysis of the core network was performed using the Metascape platform to elucidate BP, CC, MF, and signaling pathways involved. GO enrichment analysis showed that the BP involved in TSN mainly included the transmembrane receptor protein tyrosine kinase signaling pathway, regulation of protein catabolic process, Fc receptor 


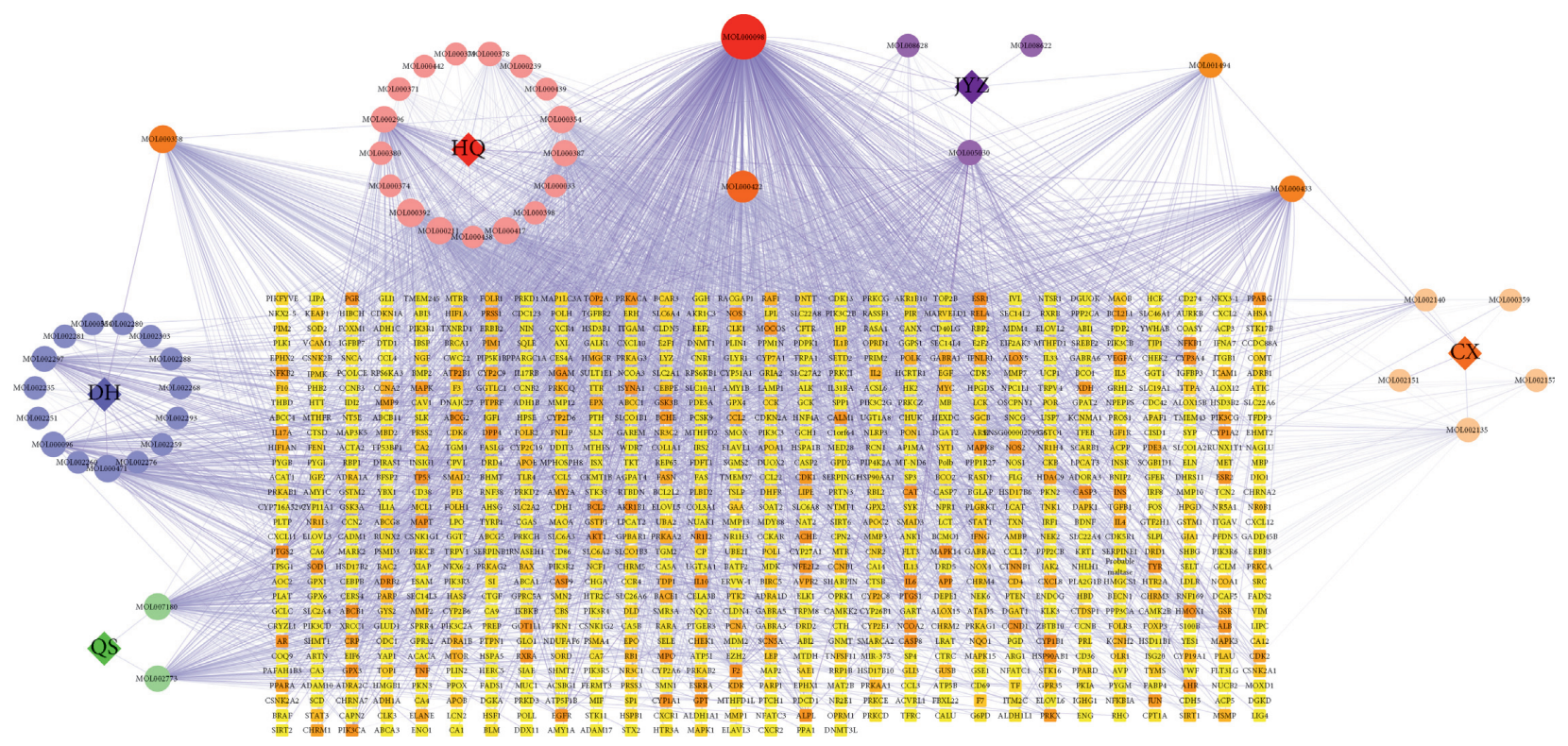

Figure 2: The Compound-Target network of TSN that consists of 910 nodes and 2912 edges. Circle and round square nodes denote the compounds and targets, respectively.

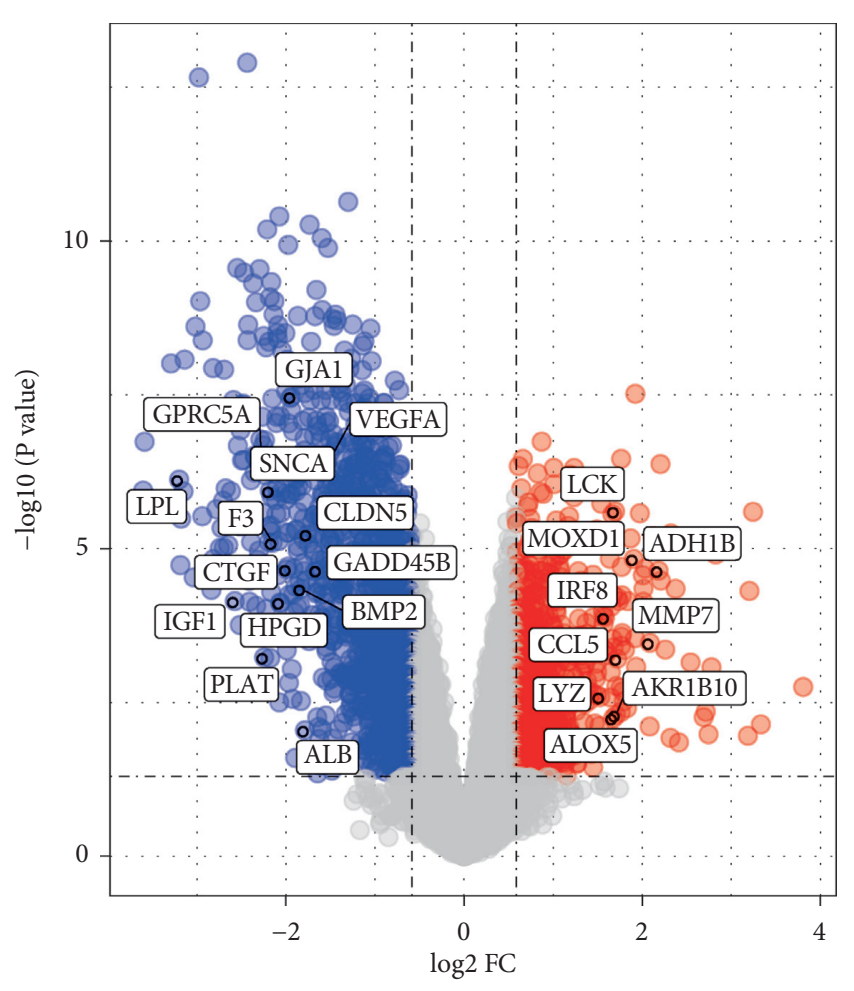

State

Down
None
Up

FIGURE 3: Volcano plot represents DEGs associated with diabetic nephropathy from GSE30528 dataset and 23 hub proteins targeted by TSN; blue plots represent downregulated DEGs; red plots represent upregulated genes.

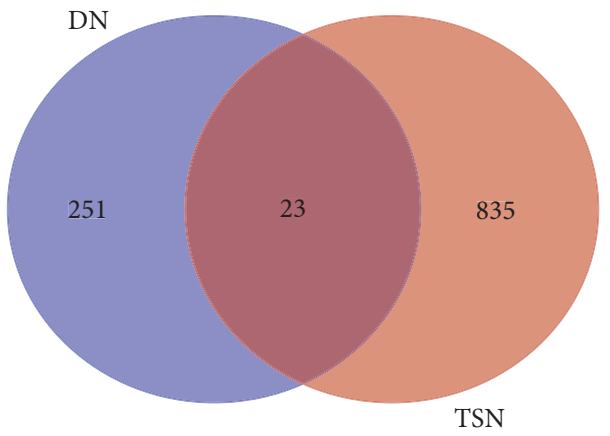

FIgURE 4: Venn diagram showing 23 TSN-related DN-DEGs.

signaling pathway, immune response-regulating signaling pathway, cellular response to growth factor stimulus, and regulation of apoptotic signaling pathway. In addition, the above BPs were associated with related MFs, including protein domain specific binding, kinase binding, ubiquitinlike protein ligase binding, protein kinase binding, phosphoprotein binding, and protein phosphorylated amino acid binding. The major CCs involved focal adhesion, cell-substrate adherent junction, cell-substrate junction, perinuclear region of the cytoplasm, membrane region, and vesicle lumen (Figure 7).

165 signaling pathways were enriched according to enrichment analysis and the first 25 are shown in Figure 8. The main related pathways included PI3K-Akt, chemokine, MAPK, focal adhesion, ErbB, estrogen, Ras, AGE-RAGE, and HIF-1, endocrine resistance, and adherent junction signaling pathway. A network was constructed using Cytoscape for visualization to uncover the relationship between targets and pathways (Figure 9). 
TABLE 2: The information of 23 TSN-related DN-DEGs.

\begin{tabular}{lcccc}
\hline Gene & Description & Log FC & $p$ value & Regulation \\
\hline LPL & Lipoprotein lipase & -3.19928 & $7.54 E-07$ & Downregulated \\
IGF1 & Insulin-like growth factor 1 & -2.57456 & $7.11 E-05$ & Downregulated \\
GPRC5A & G protein-coupled receptor class C group 5 member A & -2.27982 & $3.09 E-07$ & Downregulated \\
PLAT & Tissue-type plasminogen activator & -2.24543 & 0.000584 & Downregulated \\
SNCA & Synuclein alpha & -2.1809 & $1.15 E-06$ & Downregulated \\
F3 & Tissue factor & -2.14994 & $8.01 E-06$ & Downregulated \\
HPGD & -2.06642 & 0.000075 & Downregulated \\
CTGF & 15-Hydroxyprostaglandin dehydrogenase (NAD $(+))$ & -1.98966 & $2.17 E-05$ & Downregulated \\
GJA1 & CCN family member 2 & -1.93855 & $3.39 E-08$ & Downregulated \\
BMP2 & Gap junction alpha-1 protein & -1.82795 & $4.52 E-05$ & Downregulated \\
ALB & Bone morphogenetic protein 2 & -1.78623 & 0.00896 & Downregulated \\
CLDN5 & Albumin & -1.75802 & $5.85 E-06$ & Downregulated \\
GADD45B & Claudin 5 & -1.65127 & $2.25 E-05$ & Downregulated \\
VEGFA & Growth arrest and DNA damage inducible beta & -1.58624 & $5.92 E-07$ & Downregulated \\
LYZ & Vascular endothelial growth factor A & 1.529532 & 0.00258 & Upregulated \\
IRF8 & Lysozyme & 1.583247 & 0.000131 & Upregulated \\
ALOX5 & Interferon regulatory factor 8 & 1.673735 & 0.00516 & Upregulated \\
LCK & Arachidonate 5-lipoxygenase & 1.694745 & $2.47 E-06$ & Upregulated \\
AKR1B10 & Tyrosine-protein kinase lck & 1.705447 & 0.00508 & Upregulated \\
CCL5 & Aldo-keto reductase family 1 member B10 & 1.720493 & 0.000615 & Upregulated \\
MOXD1 & C-C motif chemokine receptor 5 & 1.905562 & $1.47 E-05$ & Upregulated \\
MMP7 & Monooxygenase 1 & 2.08645 & 0.000339 & Upregulated \\
ADH1B & Matrix metallopeptidase 7 & 2.183966 & $2.28 E-05$ & Upregulated \\
\hline
\end{tabular}

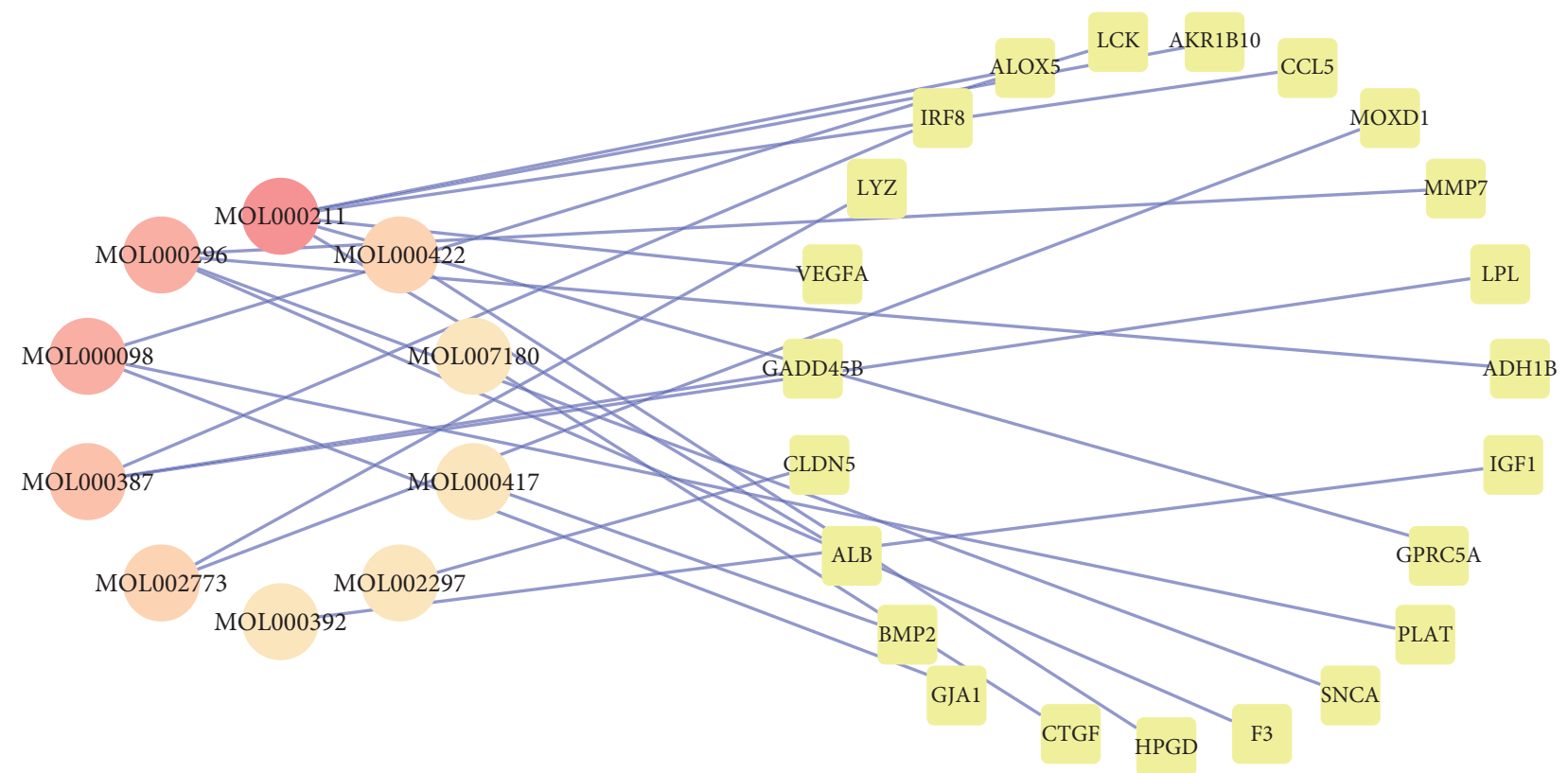

FIGURE 5: The network of compounds and 23 TSN-related DEGs. The circle and round square nodes indicate targets and compounds, respectively.

3.6. Association between DN-DEGs Related to TSN and Clinical Features of DN. The correlation between TSN-related DN-DEGs and GFR, the main clinical manifestation in DN, was investigated on the Nephroseq v5 (Supplementary Figure S2 and Table 3). There were no data for LZY and detailed information of other DEGs is shown in Supplementary Table S2. ALB, GPRC5A, PLAT, SNCA, F3, HPGD, CTGF, GJA1, BMP2, CLDN5, MODX1, GADD45B, and
VEGFA were positively correlated with GFR, suggesting that these genes contributed to renal protection. In this case, the correlation between ALB and GFR was relatively weak $(R=0.454)$. Furthermore, IRF8, ALOX5, LCK, AKR1B10, CCL5, MOXD1, MMP7, and ADH1B were negatively correlated with GFR, implying that these genes were involved in the progression of DN. However, there was a nonlinear correlation between IGF1 and GFR $(p=0.057)$. 


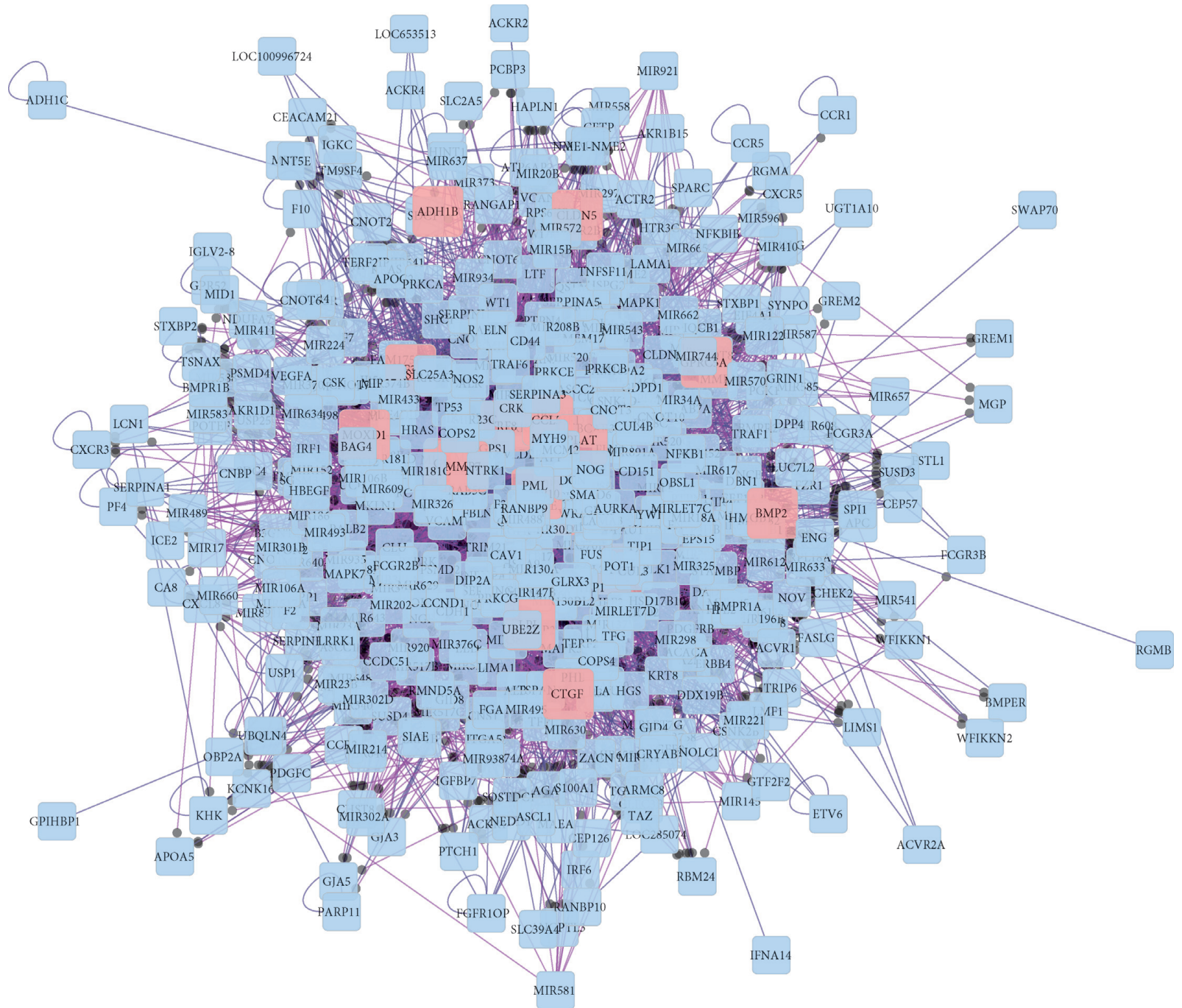

(a)

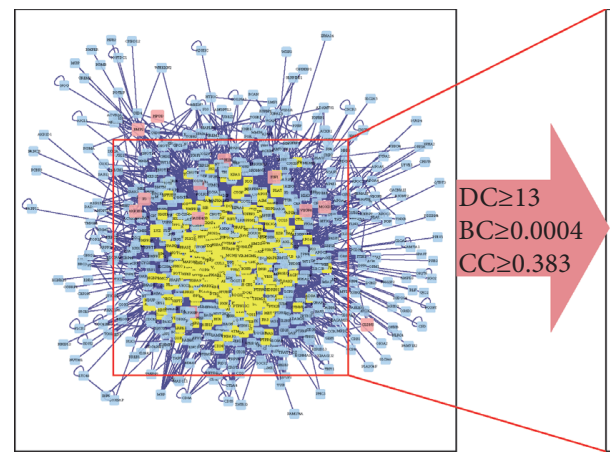

853 nodes and 9507 edges

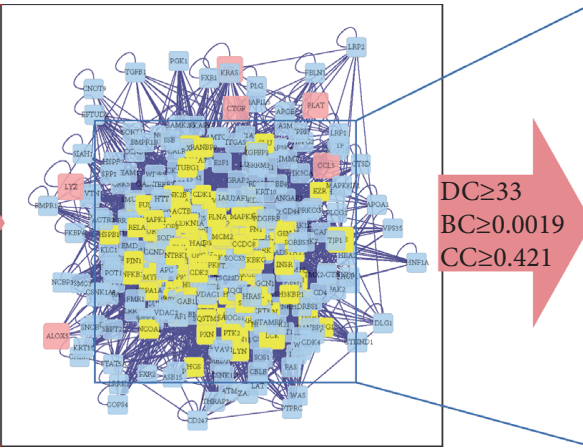

333 nodes and 5498 edges

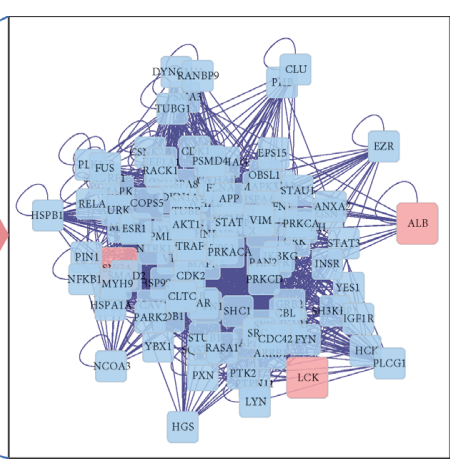

116 nodes and 1894 edges

(b)

FIgure 6: The construction and topological analysis of the PPI network. (a) The PPI network of TSN-related DN-DEGs was constructed using Bisogenet and the red nodes represent TSN-related DN-DEGs. (b) The process of topological analysis for the PPI network. 


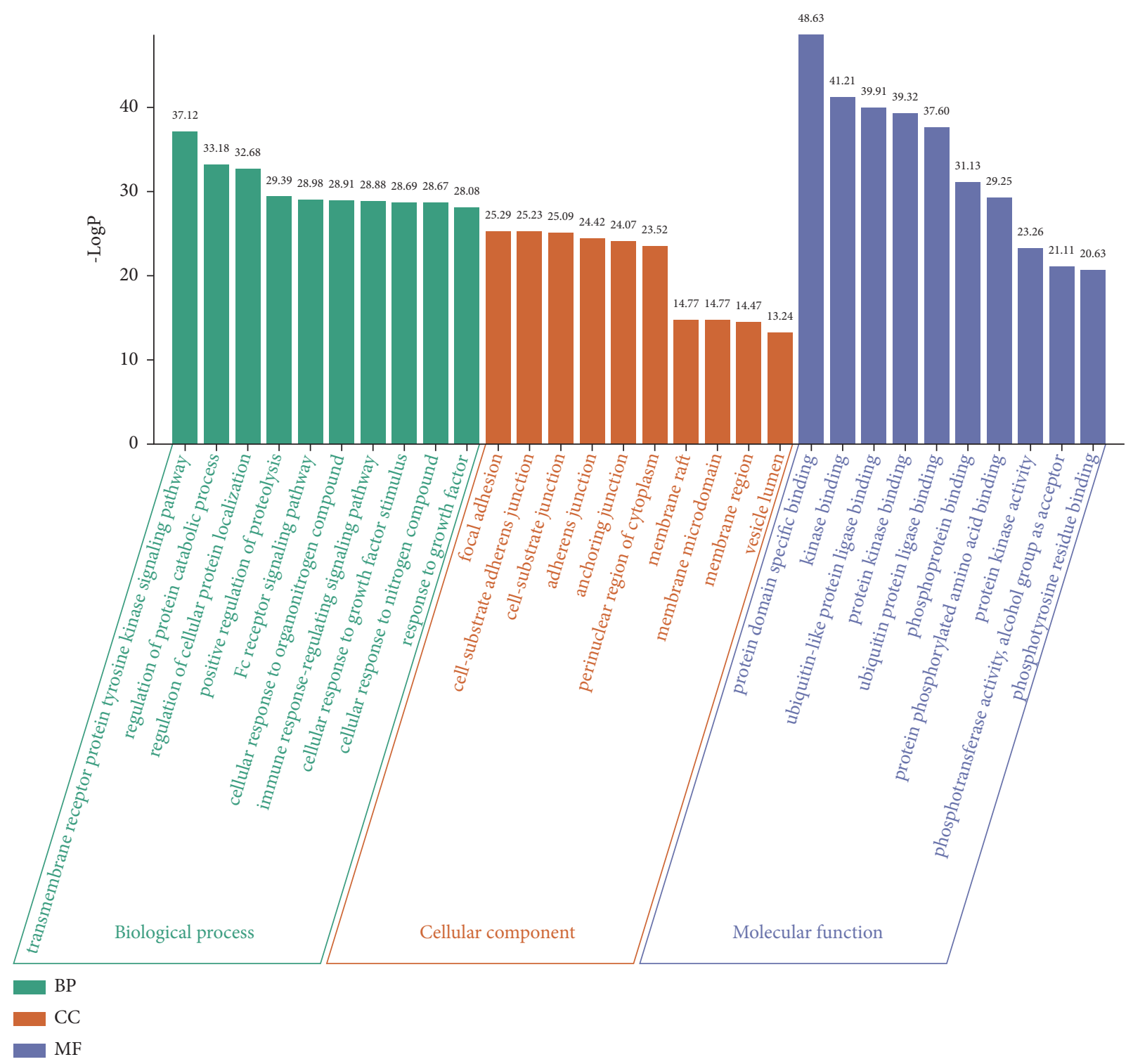

FIgURE 7: The GO enrichment of 116 key genes in PPI network. The top 10 items for each section were listed separately.

\subsection{Experimental Validation}

3.7.1. Kidney Histological Observations. HE, PAS, and Masson's staining results revealed that glomeruli from the NC group were of regular morphology, clear, and with a complete structure. Also, the mesangial membrane was not significantly thickened. There were hyperplasia and structural disorganization of glomerular cells in the DN group, with enlarged glomeruli and broadened mesangial matrix, which was accompanied by extensive collagen fibril formation and glycogen deposition. The histopathological damage in the kidney was improved in the TSN-treated rats, with relatively intact glomeruli structure and reduced fibrous deposition compared to those of $\mathrm{DN}$ animals (Figure 10). and CCL5. In order to confirm the effect of TSN treatment in TSN-related DN-DEGs, the protein levels of GJA1, CTGF, and MMP7 were evaluated by western blot, and ELISA measured renal CCL5 levels. The results showed that the levels of CTGF, MMP7, and CCL5 level were increased in the DN group, while the GJA1 were decreased compared to the NC group. Indeed, TSN treatment attenuated the increase in CCL5, MMP7, and CTGF while it upregulated GJA1 (Figure 11).

\section{Discussion}

$\mathrm{DN}$ is an important microvascular complication of DM and also the main cause of ESRD. Indeed, DN is closely associated with increased mortality in DM patients. DN is multifactorial and is characterized by decreased GFR, proteinuria, and renal ultrastructural changes. Typical pathological changes in DN include tubular and glomerular basement membrane thickening, interstitial ECM 


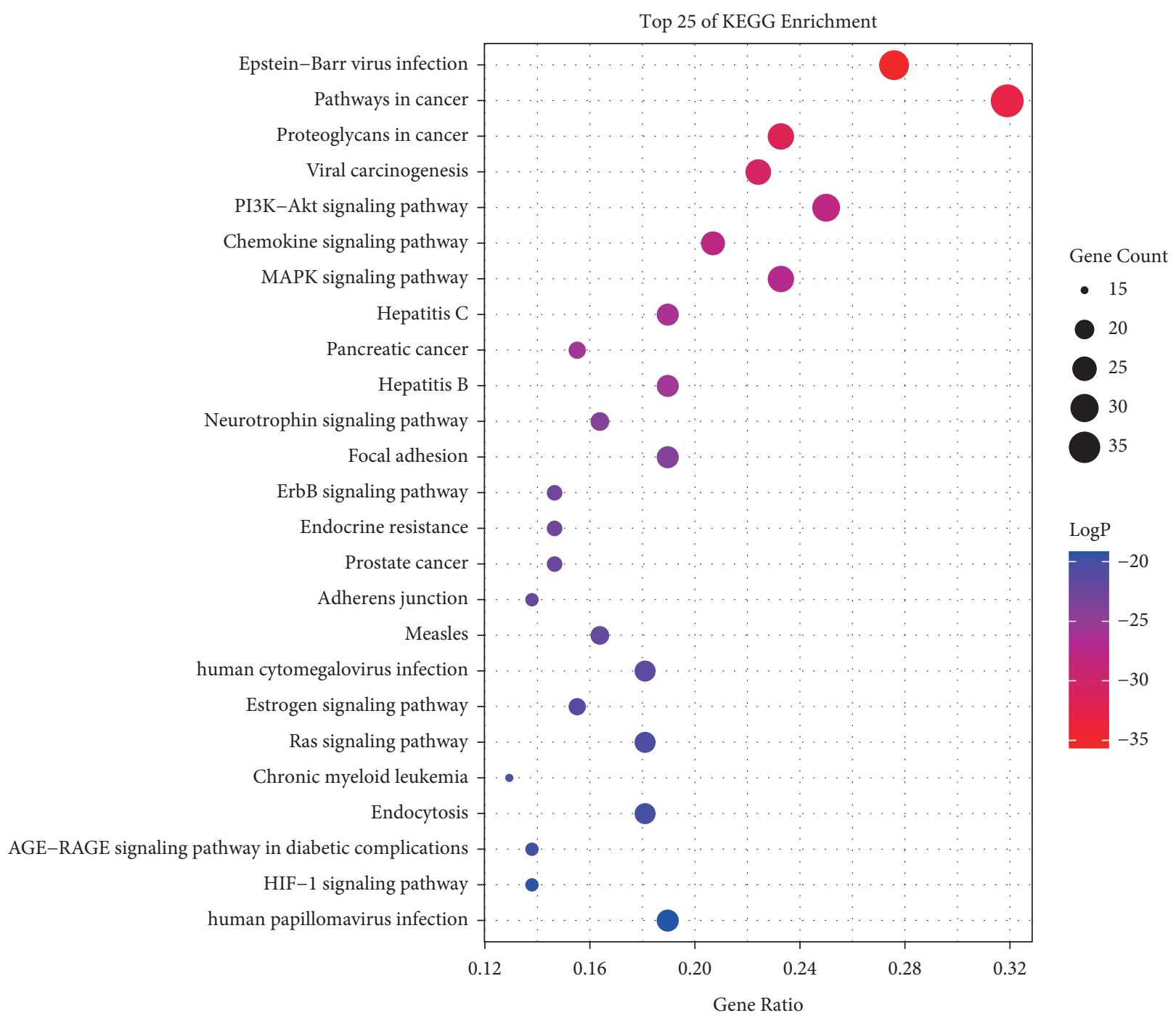

Figure 8: The KEGG enrichment analysis of the top 25 metabolic pathways.

expansion, glomerulosclerosis, and tubulointerstitial fibrosis, which ultimately cause renal hypofunction [23]. The main therapy for $\mathrm{DN}$ is to regulate blood glucose, blood lipids, and blood pressure [24]. However, the progressive decline of renal function in DN cannot be effectively prevented by glucose-lowing strategies. Therefore, the description of novel treatment strategies for $\mathrm{DN}$ is an important topic. In this regard, TCM has been used for many years in DM treatment and its complications $[7,24]$, being characterized as a multicomponent and multitarget strategy that acts on multiple pathways. TSN is an empirical formula for DN by a Chinese medicine expert, Yanbin Gao, which was effective in both humans and mice. In this study, the molecular network mechanism of TSN was explored based on network pharmacology to investigate potential underlying mechanisms of this formula used in DN.

In this study, we predicted the key herbs, active compounds, and potential effector targets in TSN. TSN-related DN-DEGs were obtained and found to be strongly correlated with decreases in GFR. GFR is an indicator of the extent of glomerular disease and the level of renal function. Therefore, these genes were identified as potential key targets of TSN treatment in DN. Besides, HuangQ $i$ and bioactive components mairin (degree $=7$ ), quercetin (degree $=5$ ), and hederagenin (degree $=5$ ) were the key components in the treatment as they were high associated with these 23 targets. Mairin, also known as betulinic acid, can reduce glucose uptake and decrease endogenous glucose production $[25,26]$ by inhibiting alpha-glucosidase activity by competitively binding to alpha-glucosidase [27]. Mairin can also inhibit NF- $\kappa$ B activation by preventing the degradation of $\mathrm{I} \kappa \mathrm{B}$ in $\mathrm{DN}$ rats, resulting in the reduction of fibrosis in $\mathrm{DN}$ [28]. Quercetin not only provides improved renal function, but also possesses effects on antihyperglycemia and insulin resistance. Furthermore, quercetin reactivates the Hippo pathway to inhibit the proliferation of glomerular mesangial cells (MCs) in DN rats and MCs treated with high glucose, while quercetin also improves renal fibrosis and renal function in $\mathrm{DN}$ [29]. In addition, quercetin was shown to improve renal function in DN rats by downregulating TGF$\beta 1$ and CTGF [30,31]. However, research on hederagenin is 


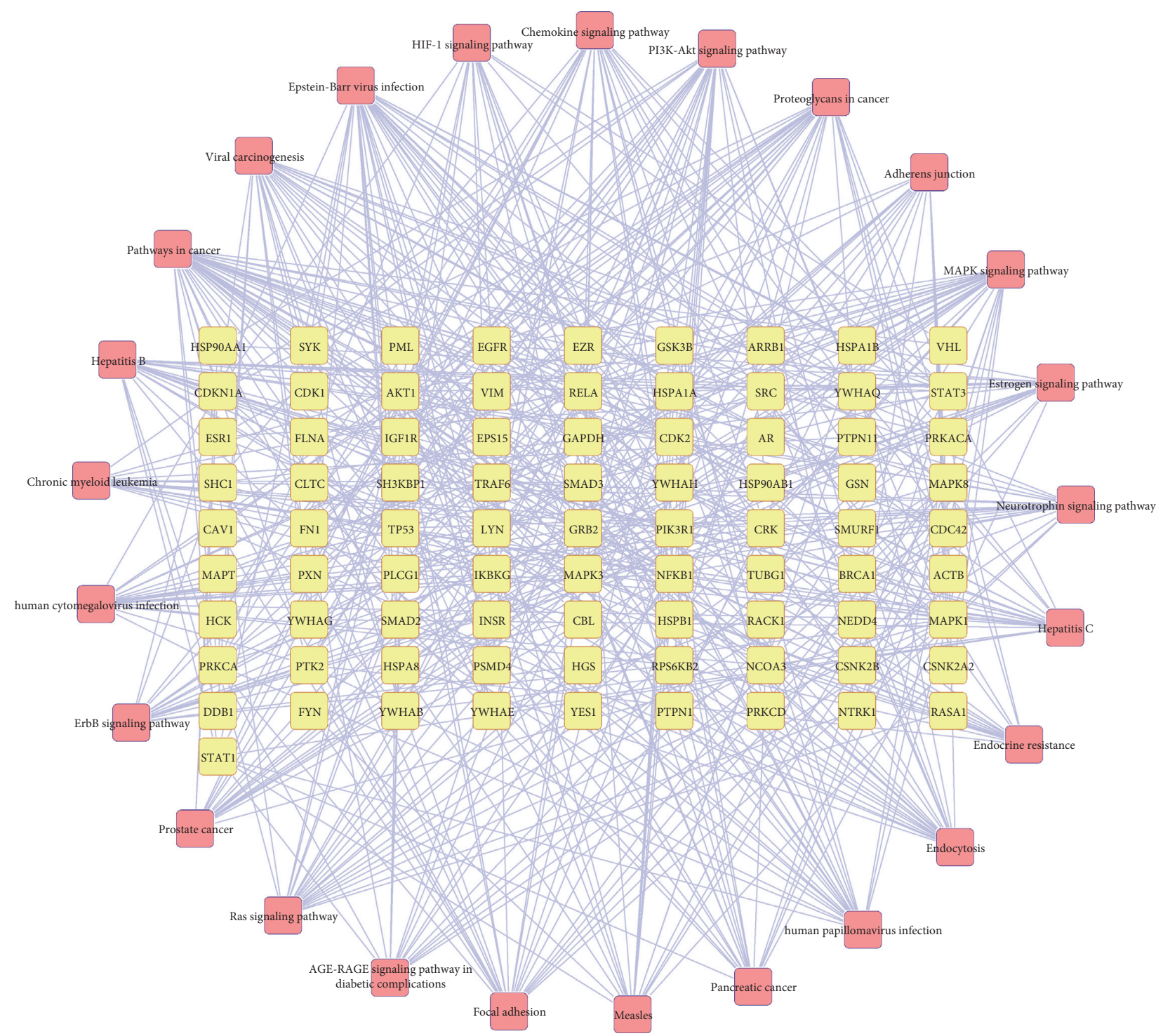

Figure 9: The network of targets involved in the major KEGG pathways. Circle and round square nodes denote the targets and signaling pathways, respectively.

relatively scarce. As a dietary fiber, it has the potential effect to reduce lipid synthesis and lipid absorption in the intestine and promote the excretion of bile acids and triglycerides [32]. Although hyperlipidemia is an important factor in DM and DN, the effects of hederagenin in DM are still unclear. Network exploration reveals TSN's multicomponent synergistic effect on DN prevention.

In order to investigate the intrinsic mechanisms of TSN, we constructed a regulatory network of TSN-related DN-DEGs and performed the GO enrichment analysis. It was suggested that TSN may influence DM progression through multiple biological processes, including growth factor regulation, immune response, cellular stress response, and apoptosis. TSN may have a regulatory effect on focal adhesion, cell-substrate adherent junction, and cell-substrate junction, and these components are mainly participating in extracellular matrix (ECM) generation.
The pathways enriched are related to ECM production, cell proliferation and migration, inflammation, and endocrine disruption and suggested that the therapeutic efficacy of TSN is mediated by the synergy of multiple pathways. For example, AGEs can upregulate RAGE expression, activate multiple signaling pathways, including JAK-STAT, MAPK/ ERK, PI3K/Akt/mTOR, and NF- $\kappa \mathrm{B}$, and increase oxidative stress, inflammation, and renal fibrosis, causing structural and functional disorders in the kidney of patients with DM [33]. Active PI3K/AKT pathway in diabetic patients upregulates CTGF [34], which is involved in ECM deposition and promotes EMT in DN $[35,36]$. Hyperglycemia also causes MAPK phosphorylation and activates the MAPK signaling pathway, the activation of which causes increased apoptosis, inflammatory cell invasion, and ECM synthesis [37]. Focal adhesion is involved in cell migration and ECM synthesis during EMT in DN [38]. Long-term overactivation of HIF$1 \alpha$ can induce the deposition in the ECM, causing 
TABLE 3: Pearson's correlations between GFR and TSN-related DN-DEGs.

\begin{tabular}{lccc}
\hline Target & $p$ value & $R$ value & $R^{2}$ \\
\hline MMP7 & $3.11 E-04$ & -0.697 & 0.485809 \\
ADH1B & $3.53 E-05$ & -0.764 & 0.583696 \\
MOXD1 & $1.59 E-04$ & -0.72 & 0.5184 \\
CCL5 & 0.001 & -0.641 & 0.410881 \\
AKR1B10 & $9.48 E-04$ & -0.655 & 0.429025 \\
LCK & $6.46 E-04$ & -0.67 & 0.4489 \\
ALOX5 & 0.007 & -0.557 & 0.310249 \\
IRF8 & 0.014 & -0.515 & 0.265225 \\
VEGFA & $2.00 E-05$ & 0.778 & 0.605284 \\
GADD45B & $5.32 E-05$ & 0.753 & 0.567009 \\
CLDN5 & $2.06 E-05$ & 0.778 & 0.454 \\
ALB & 0.034 & 0.644 & 0.206116 \\
BMP2 & 0.001 & 0.852 & 0.414736 \\
GJA1 & $4.91 E-07$ & 0.693 \\
CTGF & $3.49 E-04$ & 0.72 & 0.725904 \\
HPGD & $1.59 E-04$ & 0.817 & 0.480249 \\
F3 & $3.52 E-06$ & 0.753 & 0.5184 \\
SNCA & $5.30 E-05$ & 0.529 & 0.667489 \\
PLAT & 0.011 & 0.843 & 0.567009 \\
GPRC5A & $8.32 E-07$ & 0.412 & 0.279841 \\
IGF1 & 0.057 & 0.787 & 0.710649 \\
LPL & 0.0000137 & 0.169744 \\
\hline
\end{tabular}
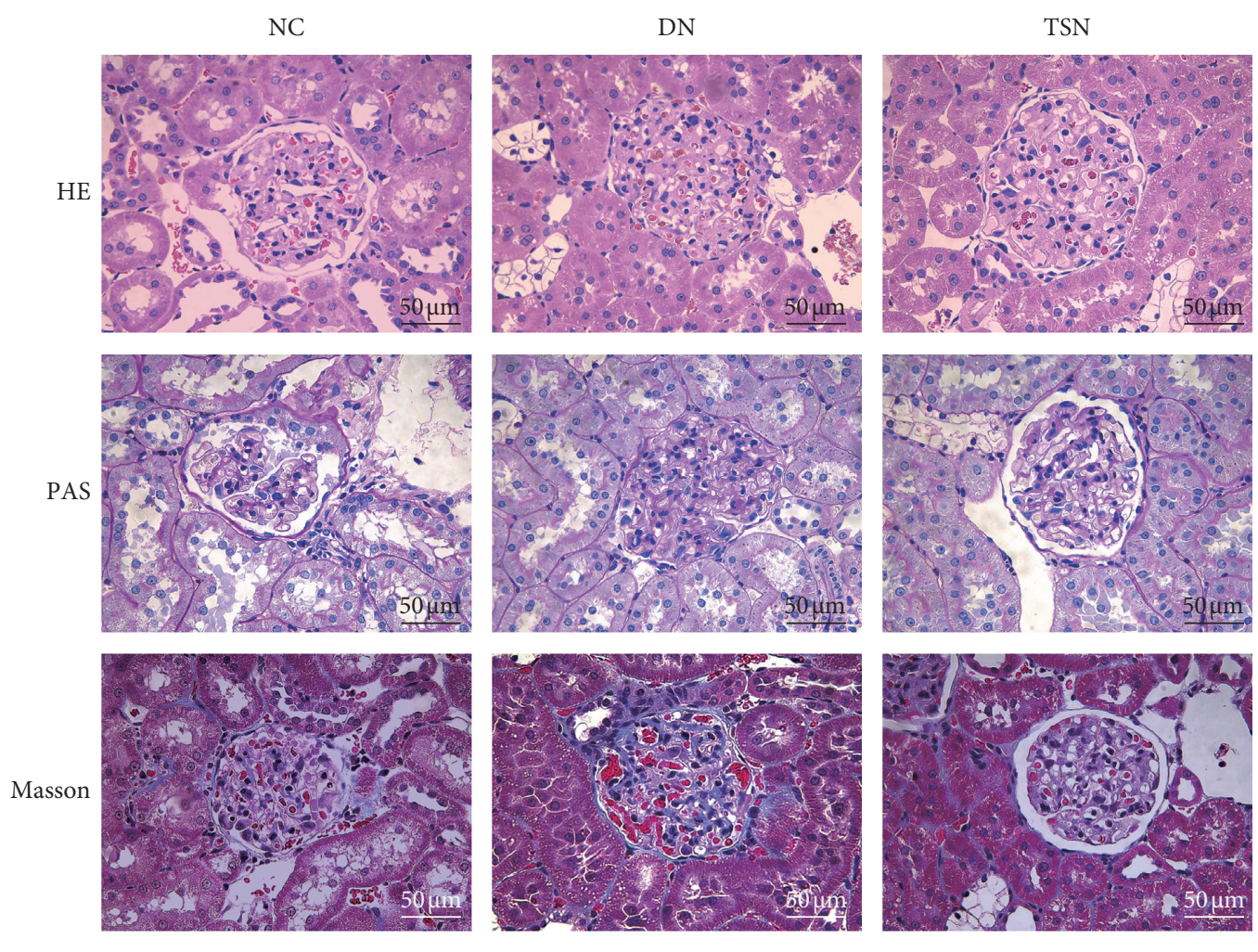

FIGURE 10: Renal histopathological changes in each group (magnification $\times 400$ ).

glomerulosclerosis and renal interstitial fibrosis [39, 40]. Previous studies have reported that TSN exhibits a nephroprotective effect in DN mice by reversing podocyte EMT [9]. Combined with the results of GO and KEGG enrichment analysis, we presumed that a potential mechanism for TSN treatment in DN may be to reduce ECM deposition in the kidneys of DN patients by modulating levels of growth factors and chemokines, improving cellular stress and inflammation, and inhibiting EMT-induced cell proliferation.

EMT is an induced transformation of damaged cells into mesenchymal cells, with metastatic and fiber-generation capacity. The EMT process generates cytokines and chemokines, accompanied by the recruitment and proliferation of fibroblasts, causing the production and deposition of 


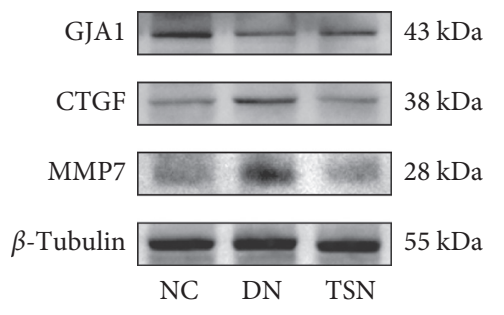

(a)
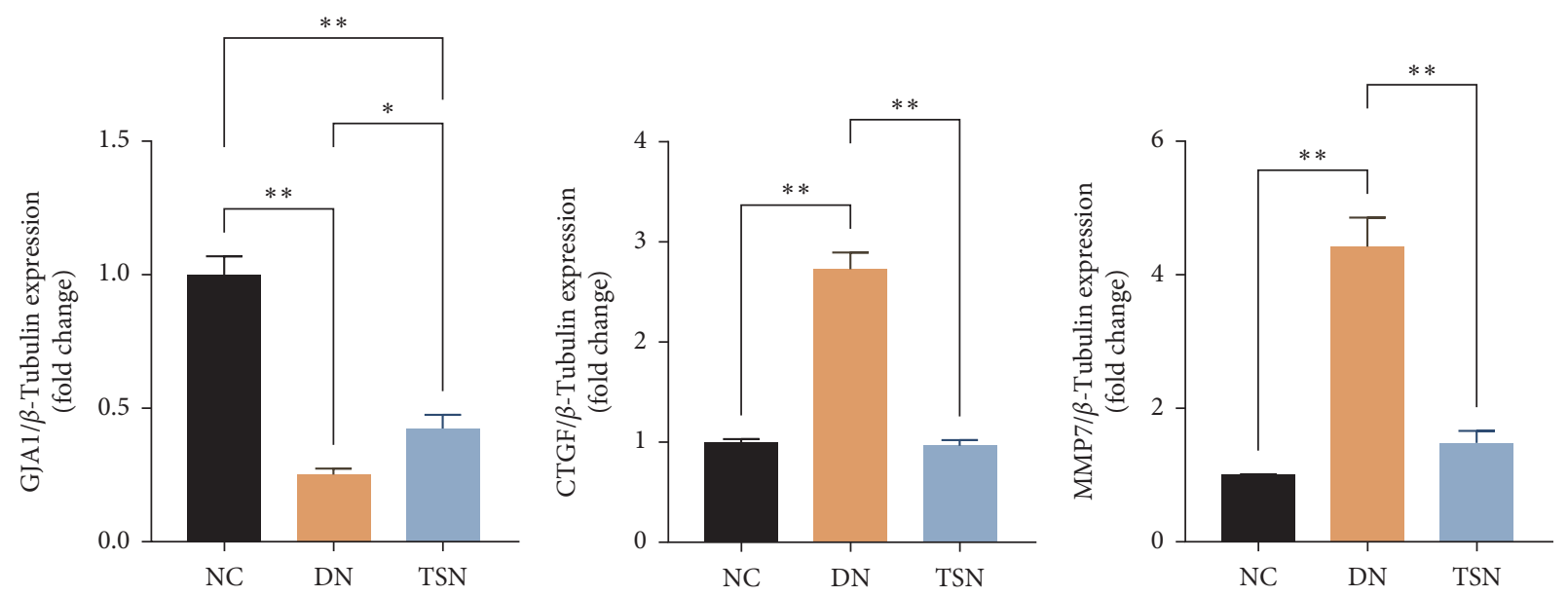

(b)

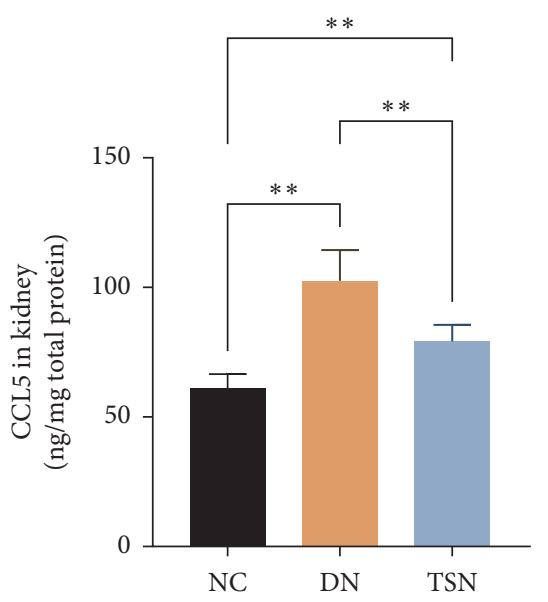

(c)

FIGURE 11: Effect of TSN on related DEGs in DN rats. Data are presented as the mean \pm SD $\left({ }^{*} P<0.05\right.$; $\left.{ }^{*} P<0.01\right)$. (a) Representative immunoblots for the CX43, CTGF, MMP7, and $\beta$-Tubulin proteins. (b) The relative expression levels of CX43/ $\beta$-Tubulin and CTGF/ $\beta$-Tubulin and MMP7/ $\beta$-Tubulin. The data on quantified protein expressions were normalized by related $\beta$-Tubulin (fold change of NC). (c) CCL5s expression in all group.

ECM, which participates in the progression of glomerulosclerosis and renal fibrosis [41-43]. Pathologically, DN presents itself with ECM accumulation and renal fibrosis. Extensive protein deposition in the basement membrane leads to thickening of the glomerular basement membrane, inducing interstitial fibrosis, glomerulosclerosis, and ultimately renal failure $[41,44]$. Therefore, therapies directed at EMT and renal fibrosis are expected to be effective strategies for the treatment of DN. Through the establishment of the DN rat model, histopathological observation revealed that
TSN effectively attenuated the extent of glomerular damage and reduced fibrous deposition, demonstrating a protective effect against renal fibrosis in DN rats.

The intrinsic mechanisms and related targets involved were further explored. Among the TSN-related DN-DEGs, GJA1, CTGF, MMP7, and CCL5 were our main targets of interest. Gap Junction Protein Alpha 1 (GJA1), also called CX43, is an essential component of cellular junctional structures during the transport of small molecules intercellularly. GJA1 prevents the proliferation of renal fibrosis in 
DN by improving oxidative stress [45] as well as downregulating TGF- $\beta 1$ levels $[46,47]$. The growth factor CTGF amplifies the fibrillogenic activity of TGF- $\beta 1$ and induces the accumulation of extracellular matrix [48, 49]. MMP7, a member of the MMP family, can cleave a series of matrix and protein fragments, and release growth factors from the extracellular matrix, while promoting renal fibrosis $[23,50,51]$. In addition, the chemokine signaling pathway was also enriched in KEGG enrichment analysis. CCL5, a member of the $\mathrm{C}$ - $\mathrm{C}$ chemokine family, exerts a powerful chemotactic effect on immune cells, which is involved in chronic inflammatory seen in progression of DN $[4,52]$. Therefore, the levels of CCL5 in each group were measured. As expected, GJA1 was upregulated and CTGF, MMP7, and CCL5 were downregulated by TSN to alleviate kidney fibrosis in $\mathrm{DN}$, and these targets were demonstrated to be engaged in the treatment.

We investigated the role of TSN for DN therapy at the molecular level using a network pharmacology method and explored the possible mechanisms of its effect. The inhibition of renal fibrosis and inflammation by TSN is predicted to be a potential mechanism for the treatment of $\mathrm{DN}$, and growth factors and chemokines may be key underlying mechanisms through which TSN exerts its nephroprotective effects.

\section{Conclusion}

In conclusion, potential active compounds, genes, and signaling pathways involved in the nephroprotection of TSN treatment were identified. TSN is perceived to be a promising strategy to treat DN through the synergistic effect of its "multicomponent, multitarget, and multipathway" compounds. Furthermore, it was verified that TSN could regulate the levels of GJA1, CTGF, MMP7, and CCL5 and alleviate renal fibrosis in DN. This work provides a science-based foundation for DN treatment with TSN. Additional experimental validation is essential to investigate other mechanisms involved in renal fibrosis and the role of the other TSN-related DEGs.

\section{Abbreviations}

DN: Diabetic nephropathy

ESRD: End-stage renal disease

TSN: Tang-Shen-Ning decoction

GFR: Glomerular filtration rate

EMT: Epithelial-mesenchymal transition

ECM: Extracellular matrix

DEGs: Differentially expressed genes

PPI: Protein-protein interaction

RAAS: Renin-Angiotensin-Aldosterone system

TCM: Traditional Chinese Medicine

HQ: $\quad$ Astragalus membranaceus (Fisch.) Bunge

QS: $\quad$ Euryale ferox Salisb. ex DC

JYZ: Rosa laevigata Michx

DH: Rheum officinale Baill

CX: Ligusticum chuanxiong Hort

OB: Oral bioavailability
DL: Drug likeness

GO: Gene Ontology

BP: Biological processes

MF: Molecular functions

CC: Cellular components.

\section{Data Availability}

The datasets used and/or analyzed during the current study are available from the corresponding author on reasonable request.

\section{Conflicts of Interest}

The authors declare no conflicts of interest.

\section{Authors' Contributions}

Y. G. and Z. Z. put forward the idea of the article and supervised the study. J. L. carried out the data preparation and network construction. J. H. established the DN model. J. L. analyzed the result and wrote the manuscript. Y. G. and Z. Z. participated and directed the revision of the manuscript. All authors read and approved the final version of the manuscript.

\section{Acknowledgments}

This research was supported by the National Key R\&D Program of China (Grant no. 2018YFC1704102).

\section{Supplementary Materials}

Supplementary material 1: Table S1. The details of DN-DEGs in GSE30528. Supplementary material 2: Table S2. The information about topological parameters in the PPI network. Supplementary material 3: Table S3. The information of GFR and TSN-related DN-DEGs provided by Nephroseq v5. Supplementary material 4: Figure S1. Gene relative expression of TSN-related DN-DEGs in GSE30528. (A) 9 upregulated targets; (B) 14 downregulated targets. Supplementary material 5: Figure S2. Correlation between TSNrelated DN-DEGs expression and GFR. (Supplementary Materials)

\section{References}

[1] R. Z. Alicic, M. T. Rooney, and K. R. Tuttle, "Diabetic kidney disease," Clinical Journal of the American Society of $\mathrm{Ne}$ phrology, vol. 12, no. 12, pp. 2032-2045, 2017.

[2] R. A. DeFronzo, W. B. Reeves, and A. S. Awad, "Pathophysiology of diabetic kidney disease: impact of SGLT2 inhibitors," Nature Reviews Nephrology, 2021.

[3] F. Persson and P. Rossing, "Diagnosis of diabetic kidney disease: state of the art and future perspective," Kidney International Supplements (2011), vol. 8, no. 1, pp. 2-7, 2018.

[4] X. Hu, W. Liu, Y. Yan et al., "Vitamin D protects against diabetic nephropathy: evidence-based effectiveness and mechanism," European Journal of Pharmacology, vol. 845, pp. 91-98, 2019. 
[5] L. F. Fried, N. Emanuele, J. H. Zhang et al., "Combined angiotensin inhibition for the treatment of diabetic nephropathy," New England Journal of Medicine, vol. 369, no. 20, pp. 1892-1903, 2013.

[6] V. Saglimbene, S. C. Palmer, M. Ruospo et al., "The long-term impact of renin-angiotensin system (RAS) inhibition on cardiorenal outcomes (LIRICO): a randomized, controlled trial," Journal of the American Society of Nephrology, vol. 29, no. 12, pp. 2890-2899, 2018.

[7] G. D. Sun, C. Y. Li, W. P. Cui et al., "Review of herbal traditional Chinese medicine for the treatment of diabetic nephropathy," Journal of Diabetes Research, vol. 2016, Article ID 5749857, 18 pages, 2016.

[8] G. Yan-bin, Z. Hui, G. Song et al., "Multicenter randomized and double-blind controlled clinical trial of Tang Shen Ning Granules in treating diabetic kidney disease," China Journal of Traditional Chinese Medicine and Pharmacy, vol. 32, no. 11, pp. 5212-5215, 2017.

[9] F. Q. Cui, Y. B. Gao, Y. F. Wang et al., "Effect of Tang-ShenNing decoction on podocyte epithelial-esenchymal transformation via inhibiting Wnt/beta-catenin pathway in diabetic mice," Annals of Palliative Medicine, 2020.

[10] R. Zhang, X. Zhu, H. Bai, and K. Ning, "Network pharmacology databases for traditional Chinese medicine: review and assessment," Frontiers in Pharmacology, vol. 10, p. 123, 2019.

[11] J. Liang, M. Wu, C. Bai et al., "Network pharmacology approach to explore the potential mechanisms of Jieduan-Niwan formula treating acute-on-chronic liver failure," EvidenceBased Complementary and Alternative Medicine, vol. 2020, Article ID 1041307, 16 pages, 2020.

[12] J. Ru, P. Li, J. Wang et al., "TCMSP: a database of systems pharmacology for drug discovery from herbal medicines," Journal of Cheminformatics, vol. 6, no. 1, p. 13, 2014.

[13] J. Liu, Y. Li, Y. Zhang et al., "A network pharmacology approach to explore the mechanisms of qishen granules in heart failure," Medical Science Monitor, vol. 25, pp. 7735-7745, 2019.

[14] S. Kim, J. Chen, T. Cheng et al., "PubChem in 2021: new data content and improved web interfaces," Nucleic Acids Research, vol. 49, no. D1, pp. D1388-d1395, 2021.

[15] D. Szklarczyk, A. Santos, C. von Mering, L. J. Jensen, P. Bork, and M. Kuhn, "STITCH 5: augmenting protein-chemical interaction networks with tissue and affinity data," Nucleic Acids Research, vol. 44, no. D1, pp. D380-D384, 2016.

[16] K. I. Woroniecka, A. S. D. Park, D. Mohtat, D. B. Thomas, J. M. Pullman, and K. Susztak, "Transcriptome analysis of human diabetic kidney disease," Diabetes, vol. 60, no. 9, pp. 2354-2369, 2011.

[17] P. Shannon, A. Markiel, O. Ozier et al., "Cytoscape: a software environment for integrated models of biomolecular interaction networks," Genome Research, vol. 13, no. 11, pp. 2498-2504, 2003.

[18] K. Xiao, K. Li, S. Long, C. Kong, and S. Zhu, "Potential molecular mechanisms of Chaihu-Shugan-San in treatment of breast cancer based on network pharmacology," Evidencebased Complementary and Alternative Medicine: eCAM, vol. 2020, Article ID 3670309, 9 pages, 2020.

[19] T. W. Valente and K. Fujimoto, "Bridging: locating critical connectors in a network," Social Networks, vol. 32, no. 3, pp. 212-220, 2010.

[20] Y. Zhou, B. Zhou, L. Pache et al., "Metascape provides a biologist-oriented resource for the analysis of systems-level datasets," Nature Communications, vol. 10, no. 1, p. 1523, 2019.
[21] J. Rowland, A. Akbarov, J. Eales et al., "Uncovering genetic mechanisms of kidney aging through transcriptomics, genomics, and epigenomics," Kidney International, vol. 95, no. 3, pp. 624-635, 2019.

[22] T. Wang, Y. Gao, X. Wang et al., "Calpain-10 drives podocyte apoptosis and renal injury in diabetic nephropathy," Diabetes, Metabolic Syndrome and Obesity: Targets and Therapy, vol. 12, pp. 1811-1820, 2019.

[23] Y. Li, L. Li, O. Zeng, J. M. Liu, and J. Yang, "H2S improves renal fibrosis in STZ-induced diabetic rats by ameliorating TGF- $\beta 1$ expression," Renal Failure, vol. 39, no. 1, pp. 265-272, 2017.

[24] R. Xue, D. Gui, L. Zheng, R. Zhai, F. Wang, and N. Wang, "Mechanistic insight and management of diabetic nephropathy: recent progress and future perspective," Journal of Diabetes Research, vol. 2017, Article ID 1839809, 7 pages, 2017.

[25] F. S. G. Silva, P. J. Oliveira, and M. F. Duarte, "Oleanolic, ursolic, and betulinic acids as food supplements or pharmaceutical agents for type 2 diabetes: promise or illusion?" Journal of Agricultural and Food Chemistry, vol. 64, no. 15, pp. 2991-3008, 2016.

[26] A. Alqahtani, K. Hamid, A. Kam et al., "The pentacyclic triterpenoids in herbal medicines and their pharmacological activities in diabetes and diabetic complications," Current Medicinal Chemistry, vol. 20, no. 7, pp. 908-931, 2013.

[27] U. Hossain, A. K. Das, S. Ghosh, and P. C. Sil, "An overview on the role of bioactive $\alpha$-glucosidase inhibitors in ameliorating diabetic complications," Food and Chemical Toxicology, vol. 145, Article ID 111738, 2020.

[28] S. Wang, Z. Yang, F. Xiong et al., "Betulinic acid ameliorates experimental diabetic-induced renal inflammation and fibrosis via inhibiting the activation of NF- $\kappa \mathrm{B}$ signaling pathway," Molecular and Cellular Endocrinology, vol. 434, pp. 135-143, 2016.

[29] D. Lei, L. Chengcheng, Q. Xuan et al., "Quercetin inhibited mesangial cell proliferation of early diabetic nephropathy through the Hippo pathway," Pharmacological Research, vol. 146, Article ID 104320, 2019.

[30] L. Bai, X. Li, L. He et al., "Antidiabetic potential of flavonoids from traditional Chinese medicine: a review," The American Journal of Chinese Medicine, vol. 47, no. 5, pp. 933-957, 2019.

[31] T. Joshi, A. K. Singh, P. Haratipour et al., "Targeting AMPK signaling pathway by natural products for treatment of diabetes mellitus and its complications," Journal of Cellular Physiology, vol. 234, no. 10, pp. 17212-17231, 2019.

[32] S. P. K. Jeepipalli, B. Du, U. Y. Sabitaliyevich, and B. Xu, "New insights into potential nutritional effects of dietary saponins in protecting against the development of obesity," Food Chemistry, vol. 318, p. 126474, 2020.

[33] D. Sanajou, A. Ghorbani Haghjo, H. Argani, and S. Aslani, "AGE-RAGE axis blockade in diabetic nephropathy: current status and future directions," European Journal of Pharmacology, vol. 833, pp. 158-164, 2018.

[34] F. Li, L. Li, M. Cheng et al., "SHIP, a novel factor to ameliorate extracellular matrix accumulation via suppressing PI3K/Akt/ CTGF signaling in diabetic kidney disease," Biochemical and Biophysical Research Communications, vol. 482, no. 4, pp. 1477-1483, 2017.

[35] Z. Xu, K. Jia, H. Wang et al., "METTL14-regulated PI3K/Akt signaling pathway via PTEN affects HDAC5-mediated epithelial-mesenchymal transition of renal tubular cells in diabetic kidney disease," Cell Death \& Disease, vol. 12, no. 1, p. 32, 2021. 
[36] M. Xue, Y. Cheng, F. Han et al., "Triptolide attenuates renal tubular epithelial-mesenchymal transition via the MiR-1885p-mediated PI3K/AKT pathway in diabetic kidney disease," International Journal of Biological Sciences, vol. 14, no. 11, pp. 1545-1557, 2018.

[37] S. Malik, K. Suchal, S. I. Khan et al., "Apigenin ameliorates streptozotocin-induced diabetic nephropathy in rats via MAPK-NF- $\kappa$ B-TNF- $\alpha$ and TGF- $\beta 1$-MAPK-fibronectin pathways," American Journal of Physiology-Renal Physiology, vol. 313, no. 2, pp. F414-F422, 2017.

[38] R. Yan, Y. Wang, M. Shi et al., "Regulation of PTEN/AKT/ FAK pathways by PPARgamma impacts on fibrosis in diabetic nephropathy," Journal of Cellular Biochemistry, 2019.

[39] X. Pang, Y. Zhang, X. Shi, Z. Peng, Y. Xing, and H. Jiarui, "Hirudin reduces the expression of markers of the extracellular matrix in renal tubular epithelial cells in a rat model of diabetic kidney disease through the hypoxia-inducible factor1alpha (HIF-1alpha)/Vascular endothelial growth factor (VEGF) signaling pathway," Medical Science Monitor, vol. 26, Article ID e921894, 2020.

[40] M. Wolf and D. A. Bushinsky, "Something old, something new, something borrowed, something black," Current Opinion in Nephrology and Hypertension, vol. 26, no. 4, pp. 241-242, 2017.

[41] N. Garcia-Fernandez, C. Jacobs-Cacha, J. M. Mora-Gutierrez, A. Vergara, J. Orbe, and M. J. Soler, "Matrix metalloproteinases in diabetic kidney disease," Journal of Clinical Medicine, vol. 9, no. 2, 2020.

[42] R. M. Carew, B. Wang, and P. Kantharidis, "The role of EMT in renal fibrosis," Cell and Tissue Research, vol. 347, no. 1, pp. 103-116, 2012.

[43] Y. Li, Q. Hu, C. Li et al., "PTEN-induced partial epithelialmesenchymal transition drives diabetic kidney disease," Journal of Clinical Investigation, vol. 129, no. 3, pp. 1129-1151, 2019.

[44] J. Sun, Y. Wang, W. Cui et al., "Role of epigenetic histone modifications in diabetic kidney disease involving renal fibrosis," Journal of diabetes research, vol. 2017, Article ID 7242384, 11 pages, 2017.

[45] X. Sun, K. Huang, X. Haiming et al., "Connexin 43 prevents the progression of diabetic renal tubulointerstitial fibrosis by regulating the SIRT1-HIF- $1 \alpha$ signaling pathway," Clinical Science, vol. 134, no. 13, pp. 1573-1592, 2020.

[46] Z. Chen, X. Xie, J. Huang et al., "Connexin43 regulates high glucose-induced expression of fibronectin, ICAM-1 and TGF$\beta 1$ via Nrf2/ARE pathway in glomerular mesangial cells," Free Radical Biology and Medicine, vol. 102, pp. 77-86, 2017.

[47] X. Xie, T. Lan, X. Chang et al., "Connexin 43 mediates NF- $\kappa$ B signalling activation induced by high glucose in GMCs: involvement of c-Src," Cell Communication and Signaling, vol. 11, no. 1, p. 38, 2013.

[48] S. G. Adler, S. Schwartz, M. E. Williams et al., "Phase 1 study of anti-CTGF monoclonal antibody in patients with diabetes and microalbuminuria," Clinical Journal of the American Society of Nephrology, vol. 5, no. 8, pp. 1420-1428, 2010.

[49] Q. Ying and G. Wu, "Molecular mechanisms involved in podocyte EMT and concomitant diabetic kidney diseases: an update," Renal Failure, vol. 39, no. 1, pp. 474-483, 2017.

[50] H. J. L. Heerspink, P. Perco, S. Mulder et al., "Canagliflozin reduces inflammation and fibrosis biomarkers: a potential mechanism of action for beneficial effects of SGLT2 inhibitors in diabetic kidney disease," Diabetologia, vol. 62, no. 7, pp. 1154-1166, 2019.
[51] M. Afkarian, L. R. Zelnick, J. Ruzinski et al., "Urine matrix metalloproteinase-7 and risk of kidney disease progression and mortality in type 2 diabetes," Journal of Diabetes and Its Complications, vol. 29, no. 8, pp. 1024-1031, 2015.

[52] S. Rayego-Mateos, J. L. Morgado-Pascual, L. Opazo-Rios et al., "Pathogenic pathways and therapeutic approaches targeting inflammation in diabetic nephropathy," International Journal of Molecular Sciences, vol. 21, no. 11, 2020. 